\title{
F-HARMONIC MEASURES, QUASIHYPERBOLIC DISTANCE AND MILLOUX'S PROBLEM
}

\author{
O. MARTIO
}

\section{Introduction}

Let $B^{2}$ be the unit disk in the plane and $C$ a relatively closed subset of $B^{2}$. If $\omega$ is the harmonic measure of $C$ with respect to the open set $B^{2} \backslash C$, then A. Beurling's projection theorem can be used to estimate $\omega$ from below. This, in turn, leads to estimates, called Milloux's problem, for bounded analytic functions $f: B^{2} \rightarrow \mathbf{C}$ which are small on the set $C$.

In this paper we consider these problems for $F$-harmonic measures $\omega$ and quasiregular mappings which are generalizations of the harmonic measure and analytic functions, respectively, to higher dimensional euclidean spaces. If $C$ is a relatively closed subset of a domain $G$ in $R^{n}$ without compact components, then in Section 2 we obtain lower bounds for $\omega(x)$ which only depend on the quasihyperbolic distance of $x$ and $C$ and on the ellipticity constant of $F$. The estimates are useful since both the $F$-harmonic measurcs and the quasihyperbolic distance are, in a sense, quasiconformal invariants. Although some of these bounds can be derived from the estimates due to V. G. Maz'ja [M], we use a new tool: Harnack's inequality for monotone super- $F$-extremals.

In Section 3 we obtain a lower bound for $\omega$ in the unit ball $B^{n}$ of $R^{n}$ provided that the set $C$ meets each sphere $\partial B^{n}(t), 0 \leqq t<1$. This estimate is based on a variation of the Carleman method introduced in [GLM 3]. The method gives essentially the same lower bound as derived in Section 2 where $C$ has no compact components in $B^{n}$. These rather elementary methods produce in $B^{n}, n \geqq 2$, lower bounds for $\omega$ which are, except for certain numerical constants, as good as lower bounds derived from Beurling's projection theorem in $B^{2}$ for the ordinary harmonic measure. Section 4 deals with Milloux's problem for quasiregular mappin£s. Milloux's problem is closely related to Hadamard's three circle theorem and its local version, based on Maz'ja's estimates, proved by S. Rickman [R], see also [V].

Throughout this paper $B^{n}(x, r)$ denotes the open $n$-ball of $R^{n}$ with center $x$ and radius $r>0$. We also use the abbreviations $B^{n}(r)=B^{n}(0, r)$ and $B^{n}=B^{n}(1)$. 


\section{F-harmonic measure and quasihyperbolic distance}

2.1. F-harmonic measure. Let $F: R^{n} \times R^{n} \rightarrow R$ be a variational kernel satisfying the usual assumptions of measurability, convexity, differentiability and homogeneity in the conformally invariant case $F(x, h) \approx|h|^{n}$, see [GLM 1, p. 48]. In particular, there are constants $0<\alpha \leqq \beta<\infty$ such that for a.e. $x \in R^{n}$

$$
\alpha|h|^{n} \leqq F(x, h) \leqq \beta|h|^{n}
$$

for all $h \in R^{n}$. Let $G$ be an open set in $R^{n}$ and let $C$ be a set in $\bar{G}$ such that $C \cap G$ is closed in $G$. We let $\omega=\omega(C, G \backslash C ; F)$ denote the $F$-harmonic measure $C$ with respect to the open set $G \backslash C$. This means that for $x \in G \backslash C$

$$
\omega(x)=\inf \{u(x): u \in \mathscr{U}\}
$$

where $\mathscr{U}$ is the upper class for $\omega$, i.e. each $u \in \mathscr{U}$ is a non-negative super- $F$-extremal in $G \backslash C$ and for each $y \in \partial(G \backslash C)$

$$
\varliminf_{x \rightarrow y} u(x) \geqq \chi_{c}(y)
$$

where $\chi_{C}$ is the characteristic function of $C$. For the definition and properties of $\omega$ see [GLM 2] and [GLM 3, 2.7]. Here we are mainly interested in the case where $C$ is a closed subset of a domain $G$.

For the next lemma we recall that a continuous function in an open set $G$ is monotone if for all domains $D$ with compact closure in $G$

$$
\operatorname{osc}(u, D)=\operatorname{osc}(u, \partial D)
$$

where $\operatorname{osc}(u ; A)=\sup _{A} u-\inf _{A} u$.

2.3. Lemma. Suppose that $u$ is a continuous, monotone and non-negative superF-extremal in $G$. Then

$$
\sup _{x \in B(r / 2)} u(x) \leqq e^{c(\beta / \alpha)^{1 / n}} \inf _{x \in B(r / 2)} u(x)
$$

whenever the ball $B(r)=B^{n}\left(x_{0}, r\right)$ lies in $G$. The constant $c$ depends only on $n$.

Proof. The proof is similar to [GLM 1, Theorem 4.15], however, the details are different. Fix a ball $B(r)=B^{n}\left(x_{0}, r\right)$ in $G$. We may assume that $\bar{B}(r) \subset G$. We may also assume that $u>0$ in $\bar{B}(r)$ since if $u(x)=0$ at some point $x \in \bar{B}(r)$, then it easily follows from the $F$-comparison principle and from the continuity of $u$ that $u \equiv 0$ in a neighborhood of $\bar{B}(r)$ and hence the inequality (2.4) would be trivial.

Next let $v=\log u$ in $B(r)$. It follows from [LM, Lemma 2.12] that for each $0<\varrho<r$

$$
\int_{B(\varrho)}|\nabla v|^{n} d m \leqq c_{1}(\beta / \alpha)(\log (r / \varrho))^{1-n}
$$


where the constant $c_{1}$ depends only on $n$. To complete the proof for (2.4), note that since $u$ is monotone, $v$ is also monotone and [GLM 1, Lemma 2.7] yields for $\varrho=r / \sqrt{2}$

$$
\begin{gathered}
\omega(v, B(r / 2))^{n} \log (2 \varrho / r) \leqq \int_{r / 2}^{\varrho} \omega(v, B(t))^{n} / t d t \\
=\int_{r / 2}^{\varrho} \omega(v, \partial B(t))^{n} / t d t \leqq A \int_{B(\varrho)}|\nabla v|^{n} d m \leqq A c_{1}(\beta / \alpha)(\log (r / \varrho))^{1-n}
\end{gathered}
$$

where (2.5) has been used in the last step and $A$ depends only on $n$. This implies

$$
\log \frac{\sup u}{\inf u}=\omega(v, B(r / 2)) \leqq\left[A c_{1}(\beta / \alpha)\right]^{1 / n}(\log \sqrt{2})^{-1}
$$

and we obtain (2.4) with

$$
c=\left(A c_{1}\right)^{1 / n}(\log \sqrt{2})^{-1} .
$$

The Harnack's inequality of Lemma 2.3 can be used for $F$-harmonic measures in the following situation.

2.6. Lemma. Suppose that $C$ is a relatively closed subset of $G$ without compact components. Let $\omega^{*}(x)=\omega(C, G \backslash C ; F)(x)$ for $x \in G \backslash C$ and $\omega^{*}(x)=1$ for $x \in C$. Then $\omega^{*}$ is continuous in $G$ and (2.4) holds for $\omega^{*}$ in each ball $B^{n}\left(x_{0}, r\right) \subset G$.

Proof. The continuity of $\omega^{*}$ in $G$ follows from [GLM 3, Remark 2.14]. Next the $F$-comparison principle implies that $\omega^{*}$ is a super- $F$-extremal in $G$ and since $0<\omega^{*} \leqq 1$ and since each point $x \in G$ where $\omega^{*}(x)=1$ belongs to a continuum reaching to $\partial G, \omega^{*}$ is monotone in $G$. Thus the inequality (2.4) for $\omega^{*}$ follows from Lemma 2.3.

2.7. Quasihyperbolic distance. Suppose that $G$ is a proper subdomain of $R^{n}$. The quasihyperbolic metric $k_{G}$ in $G$ is defined as

$$
k_{G}\left(x_{1}, x_{2}\right)=\inf _{\gamma} \int_{\gamma} \operatorname{dist}(x, \partial G)^{-1} d s
$$

where the infimum is taken over the family of all rectifiable curves $\gamma$ in $G$ joining $x_{1}$ to $x_{2}$. For the properties of $k_{G}$ see [GP]. If $C$ is a subset of $G$, then for $x \in G$

$$
k_{G}(x, C)=\inf _{y \in C} k_{G}(x, y)
$$

denotes the quasihyperbolic distance of $x$ and $C$. If $C=\emptyset$, we set $k_{G}(x, C)=\infty$.

2.9. Theorem. Suppose that $G$ is a proper subdomain of $R^{n}$ and $C$ is a relatively closed subset of $G$ without compact components. Then for each $x \in G \backslash C$

$$
\omega(x) \geqq b \exp \left(-a k_{G}(x, C)\right)
$$

where $\omega=\omega(C, G \backslash C ; F)$ and $a=c(\beta / \alpha)^{1 / n}$. The constant $c>0$ depends only on $n$ and $b>0$ depends only on $n$ and $\alpha / \beta$. 
Proof. Fix $x \in G \backslash C$ and set $\delta=k_{G}(x, C)$. If $\delta=\infty$, then there is nothing to prove. Suppose $0<\delta<\infty$ and let $0<\varepsilon<\delta / 2$. Pick $y \in C$ and a rectifiable curve $\gamma$ in $G$ joining $x$ to $y$ with

$$
\delta=k_{G}(x, C)>\int_{\gamma} \operatorname{dist}(z, \partial G)^{-1} d s-\varepsilon .
$$

Next choose points $z_{1}, \ldots, z_{j+1}$ on $\gamma$ and radii $r_{1}, \ldots, r_{j}$ inductively as follows. Set $z_{1}=y$ and $r_{1}=\operatorname{dist}\left(z_{1}, \partial G\right) / 2$. Assume that $z_{1}, \ldots, z_{i}$ have been chosen and let $\gamma_{i}$ denote the part of $\gamma$ from $z_{i}$ to $x$. If $\gamma_{i} \subset \bar{B}^{n}\left(z_{i}, r_{i}\right)$, then set $j=i$ and $z_{j+1}=x$. If $\gamma_{i} \triangleleft \bar{B}\left(z_{i}, r_{i}\right)$, then let $z_{i+1}$ be the last point where $\gamma_{i}$ meets $\partial B^{n}\left(z_{i}, r_{i}\right)$ and put $r_{i+1}=\operatorname{dist}\left(z_{i+1}, \partial G\right) / 2$. Since $\gamma$ is a compact subset of $G$, the process ends after a finite number of steps.

Fix $i=1, \ldots, j-1$. Let $\gamma_{i}$ be the part of $\gamma$ from $z_{i}$ to $z_{i+1}$. Pick $z^{\prime} \in \partial G$ such that

$$
2 r_{i}=\operatorname{dist}\left(z_{i}, \partial G\right)=\left|z_{i}-z^{\prime}\right| .
$$

Then for $z \in \gamma_{i} \cap B^{n}\left(z_{i}, r_{i}\right)$

and thus

$$
\operatorname{dist}(z, \partial G) \leqq\left|z-z^{\prime}\right| \leqq\left|z-z_{i}\right|+\left|z_{i}-z^{\prime}\right| \leqq r_{i}+2 r_{i}=3 r_{i}
$$

Hence

$$
\begin{aligned}
\int_{\gamma_{i}} \operatorname{dist}(z, \partial G)^{-1} d s & \geqq \int_{\gamma_{i} \cap B^{n}\left(z_{i}, r_{i}\right)} \operatorname{dist}(z, \partial G)^{-1} d s \\
& \geqq r_{i} / 3 r_{i}=1 / 3 .
\end{aligned}
$$

$$
\int_{\gamma} \operatorname{dist}(z, \partial G)^{-1} d s \geqq \sum_{i=1}^{j-1} \int_{\gamma_{i}} \operatorname{dist}(z, \partial G)^{-1} d s \geqq(j-1) / 3
$$

and by (2.11)

This yields

$$
\delta>(j-1) / 3-\varepsilon>(j-1) / 3-\delta / 2 .
$$

$$
k_{G}(x, C)=\delta>(j-1) / 6 .
$$

Next let $\omega^{*}$ be defined as in Lemma 2.6. We apply (2.4) to $\omega^{*}$ in each $B_{i}=$ $B^{n}\left(z_{i}, r_{i}\right), i=1, \ldots, j$. Note that $B^{n}\left(z_{i}, 2 r_{i}\right) \subset G$ and hence

where

$$
\begin{gathered}
1=\omega^{*}(y)=\omega^{*}\left(z_{1}\right) \leqq \lambda \inf _{\boldsymbol{B}_{1}} \omega^{*} \leqq \lambda \sup _{\boldsymbol{B}_{\mathbf{2}}} \omega^{*} \leqq \lambda^{2} \inf _{\boldsymbol{B}_{\mathbf{2}}} \omega^{*} \\
\leqq \ldots \leqq \lambda^{j} \inf _{\boldsymbol{B}_{j}} \omega^{*} \leqq \lambda^{j} \omega^{*}(x) \leqq \lambda^{6 \delta+1} \omega(x)
\end{gathered}
$$

$$
\lambda=\exp \left(c(\beta / \alpha)^{1 / n}\right)
$$

and $c$ depends only on $n$. This implies the required inequality (2.10) with

$$
a=6 \log \lambda=6 c(\beta / \alpha)^{1 / n}, \quad b=1 / \lambda=\exp \left(-c(\beta / \alpha)^{1 / n}\right) .
$$

2.12. Remark. The use of the Harnack inequality to estimate harmonic functions or $F$-extremals is well-known, see e.g. [GLM 2] and [V 2].

The next lemma, needed in Section 3, shows that Theorem 2.9 can be used in some cases to estimate $\omega$ although $C$ is not a subset of $G$. 
2.13. Lemma. Let $G$ be the annulus $B^{n} \backslash \bar{B}^{n}\left(r_{0}\right), 0<r_{0}<1$, and $C=\partial B^{n}\left(r_{0}\right)$. Then for each $x \in G$

$$
\omega(x) \geqq\left\{\begin{array}{lll}
b\left(r_{0}(1-|x|)\right)^{a}, & \text { if } & r_{0} \leqq 1 / 2, \\
b\left(\left(1-r_{0}\right)^{-1}(1-|x|)\right)^{a}, & \text { if } & r_{0}>1 / 2,
\end{array}\right.
$$

where $\omega=\omega(C, G ; F)$ and $a, b$ are the constants of Theorem 2.9 depending only on $n$ and $\alpha / \beta$.

Proof. Fix $x \in G$. Without loss of generality we may assume $x=t e_{n}$ where $e_{n}$ is the $n$-th unit vector of $R^{n}$. Let $H$ denote the upper half space of $R^{n}$ and write $G^{\prime}=$ $H \cap B^{n}$ and $C^{\prime}=H \cap \bar{B}^{n}\left(r_{0}\right)$. Then $C^{\prime}$ is a relatively closed subset of $G^{\prime}$. If $\omega^{\prime}$ is the $F$-harmonic measure of $C^{\prime}$ with respect to $G^{\prime}$, then Carleman's principle [GLM 3, Lemma 2.8] implies $\omega^{\prime} \leqq \omega$ in $G^{\prime} \backslash C^{\prime}$ and it remains to find the lower bounds in (2.14) for $\omega^{\prime}$.

By Theorem 2.9

$$
\omega^{\prime}(x) \geqq b \mathrm{e}^{-a k(x)}
$$

where $k(x)=k_{G^{\prime}}\left(x, C^{\prime}\right)$. Let $\gamma$ be the line segment from $r_{0} e_{n}$ to $x$. Then

$$
k(x) \leqq \int_{\gamma} \operatorname{dist}\left(z, \partial G^{\prime}\right)^{-1} d s=\int_{r_{0}}^{t} \min (1-s, s)^{-1} d s
$$

and the last integral has the following values:

(i) $-\log \left(4 r_{0}(1-t)\right)$ for $r_{0} \leqq 1 / 2<t$,

(ii) $\log \left(\left(1-r_{0}\right) /(1-t)\right)$ for $1 / 2 \leqq r_{0}<t$, and

(iii) $\log \left(t / r_{0}\right) \quad$ for $r_{0}<t \leqq 1 / 2$.

Since for $0<t \leqq 1 / 2, t \leqq(4(1-t))^{-1}$ and hence the upper bound of (i) for $k(x)$ can be used in the case (iii) as well. Thus (2.15) and (2.16) yield the required estimate (2.14).

2.17. Remarks. (a) Let $B^{n-1}$ be the open unit ball of $R^{n-1}$ and let $G=B^{n-1} \times R$ be a circular infinite cylinder in $R^{n}$. Set $C=B^{n-1} \times\{0\}$ and fix $x=(0, \ldots, 0, t) \in G$ where $t>0$. Then $k_{G}(x, C) \leqq t$ and hence Theorem 2.9 implies

$$
\omega(x) \geqq b \exp (-a t) .
$$

On the other hand, it was shown in [HM, Theorem 3.35] that

$$
\omega(x) \leqq M \exp \left(-a_{1} t\right)
$$

where $M<\infty$ is an absolute constant and $a_{1}>0$ depends only on $n$ and $\alpha / \beta$. Hence Theorem 2.9 is essentially the best possible.

(b) It is not possible to choose $b=1$ in Theorem 2.9. For ordinary plane harmonic measure this follows from Theorem 2.24 below. 
2.18. Theorem. Let $G, C$ and $\omega$ be as in Theorem 2.9. Then for $x \in G \backslash C$

$$
\omega(x) \geqq 1-c k_{G}(x, C)^{\delta}
$$

where the constants $c>0$ and $\delta \in(0,1)$ depend only on $n$ and $\alpha / \beta$.

Proof. First extend $\omega$ to $\omega^{*}: G \rightarrow R$ as in Lemma 2.6. Then $\omega^{*} \in C(G) \cap$ $\operatorname{loc} W_{n}^{1}(G)$ is a monotone super-F-extremal in $G$. Next we can use the proof of Lemma 4.2 in [GLM 1] to obtain

$$
\int_{B^{n}\left(x_{0}, r\right)}\left|\nabla \omega^{*}\right|^{n} d m \leqq c_{1}(\beta / \alpha) \operatorname{osc}\left(\omega^{*}, B^{n}\left(x_{0}, \varrho\right)\right)^{n}(\ln (\varrho / r))^{1-n}
$$

where $0<r<\varrho$ and $B^{n}\left(x_{0}, \varrho\right) \subset G$. Here $c_{1}$ depends only on $n$. In fact, the proof given in [GLM 1] can directly be used for sub-F-extremals via the important extremality property [GLM 1 , Theorem 5.17 , (ii)] of regular sub- $F$-extremals. Since a function $u$ is a super- $F$-extremal if and only if $-u$ is a sub- $F$-extremal, the proof also gives (2.20).

Next the proof of Theorem 4.7 in [GLM 1] together with the inequality (2.20) and the monotonicity of $\omega^{*}$ yields

$$
\operatorname{osc}\left(\omega^{*}, B^{n}\left(x_{0}, r\right)\right) \leqq e(r / \varrho)^{\delta}
$$

where $e$ is Neper's number and $\delta=c_{2}(\alpha / \beta)^{1 / n}>0$. Here $c_{2}$ depends only on $n$ and $0<r<\varrho$ with $B^{n}\left(x_{0}, \varrho\right) \subset G$.

To prove (2.19) we may assume that $C \neq \emptyset$. Fix $x \in G \backslash C$ and suppose first that

Pick $y \in C$ such that

$$
k(x)=k_{G}(x, C)<1 / e .
$$

By [GP, Lemma 2.1]

$$
k_{G}(x, y) \leqq \min (2 k(x), 1 / e) .
$$

$$
k_{G}(x, y) \geqq \log \left(1+\frac{|x-y|}{\operatorname{dist}(y, \partial G)}\right),
$$

and since $\log (1+t) \geqq t / 2$ for $0 \leqq \log (1+t) \leqq 1 / e$, we obtain

$$
2 k(x) \geqq k_{G}(x, y) \geqq \frac{|x-y|}{2 \operatorname{dist}(y, \partial G)} .
$$

Set $\varrho=\operatorname{dist}(y, \partial G)$ and $r=|x-y|$. Then (2.21) yields

$$
\omega^{*}(y)-\omega^{*}(x) \leqq \operatorname{osc}\left(\omega^{*}, B^{n}(y, r)\right) \leqq e(r / \varrho)^{\delta} \leqq e 4^{\delta} k(x)^{\delta}
$$

and since $\omega^{*}(y)=1$ and $\omega^{*}(x)=\omega(x)$, we obtain the desired estimate (2.19) with $c=e 4^{\delta}$.

If $k(x) \geqq 1 / e$, then

$$
1-e 4^{\delta} k(x)^{\delta} \leqq 1-e 4^{\delta} e^{-\delta} \leqq 1-e 4^{\delta} e^{-1} \leqq 0
$$

and hence we again obtain (2.19) with the same $c$ and $\delta$ as above. 
2.22. Remark. The above proof yields

$$
\omega(x) \geqq 1-e(|x-y| / \operatorname{dist}(y, \partial G))^{\delta}
$$

for each $x \in G \backslash C$ and $y \in C$.

2.23. Corollary. Let $G, C$ and $\omega$ be as in Theorem 2.9. Then for each $x \in G \backslash C$

$$
\omega(x) \geqq \max \left(1-c k_{G}(x, C)^{\delta}, b \exp \left(-a k_{G}(x, C)\right)\right)
$$

where the positive constants $a, b, c$ and $\delta$ depend only on $n$ and $\alpha / \beta$.

For the ordinary plane harmonic measure A. Beurling's projection theorem gives a more precise result than (2.19).

2.24. Theorem. Let $G$ be a plane domain and $C$ a relatively closed subset of $G$ without compact components. If $\omega$ is the ordinary harmonic measure of $C$ with respect to $G \backslash C$, then for $x \in G \backslash C$

$$
\omega(x) \geqq 1-\frac{8}{\pi} k_{G}(x, C)^{1 / 2} .
$$

The exponent $1 / 2$ is best possible.

Proof. As in the proof of Theorem 2.18 let $x \in G \backslash C$. Let $\varepsilon>0$ and choose $y \in C$ such that

$$
k_{G}(x, y)<k_{G}(x, C)+\varepsilon .
$$

Assume first that $k_{G}(x, y)<1 / e$. Then, see the proof for Theorem 2.18,

$$
k_{G}(x, y) \geqq \log \left(1+\frac{|x-y|}{\operatorname{dist}(y, \partial G)}\right) \geqq \frac{1}{2} \frac{|x-y|}{\operatorname{dist}(y, \partial G)} .
$$

Let $r=|x-y|$ and $\varrho=\operatorname{dist}(y, \partial G)$. For the rest of the proof we may assume $y=0$, $x=r e_{1}$ and $\varrho=1$. Let $\omega^{\prime}$ be the ordinary harmonic measure of $C \cap B^{2}$ with respect to $B^{2} \backslash C$. By Beurling's projection theorem $[\mathrm{B}], \omega^{\prime}(x) \geqq \omega^{*}(x)$ where $\omega^{*}$ is the harmonic measure of $C^{*}=\left(-e_{1}, 0\right]$ with respect to $B^{2} \backslash C^{*}$. On the other hand, it is well known that for each $0<t<1$

$$
\omega^{*}\left(t e_{1}\right)=1-\frac{4}{\pi} \arctan \sqrt{t}
$$

By Carleman's principle, $\omega(x) \geqq \omega^{\prime}(x)$ and hence the above inequalities yield

$$
\begin{aligned}
& \omega(x) \geqq \omega^{\prime}(x) \geqq \omega^{*}(x)=1-\frac{4}{\pi} \arctan \sqrt{r} \geqq 1-\frac{4}{\pi} \sqrt{r} \\
& \geqq 1-\frac{4 \sqrt{2}}{\pi} k_{G}(x, y)^{1 / 2} \geqq 1-\frac{8}{\pi}\left(k_{G}(x, C)+\varepsilon\right)^{1 / 2} .
\end{aligned}
$$

Letting $\varepsilon \rightarrow 0$ we obtain the desired result in the case $k_{G}(x, C)<1 / e$.

If $k_{G}(x, C) \geqq 1 / e$, then $8 /(\pi \sqrt{e})>1$ and the estimate $(2.25)$ is trivial. 
Letting $\omega=\omega^{*}$ where $\omega^{*}$ is as above we see from (2.26) that the constant $1 / 2$ cannot be replaced by any constant $\delta>1 / 2$.

2.27. Remark. Let $G, C$ and $\omega$ be as in Theorem 2.24. It follows from the above proof that

$$
\omega(x) \geqq 1-\frac{4}{\pi}(|x-y| / \operatorname{dist}(y, \partial G))^{1 / 2}
$$

and $4 / \pi$ and $1 / 2$ cannot be replaced by any smaller and bigger constant, respectively.

\section{Estimates in a ball}

The following theorem is a counterpart of an estimate due to T. Carleman [C] and A. Beurling [B] for $F$-harmonic measures. The proof is based on the proof of Phragmen-Lindelöf's principle in [GLM 3].

3.1. Theorem. Suppose that $C$ is a closed subset of the unit ball $B^{n}$ with the property that the spheres $\partial B^{n}(t)$ meet $C$ for all $0 \leqq t<1$. Let $\omega$ be the F-harmonic measure of $C$ with respect to $B^{n} \backslash C$. Then for each $x \in B^{n} \backslash C$

$$
\omega(x) \geqq c_{1}(1-|x|)^{a}
$$

where $c_{1}>0$ depends only on $n$ and $\alpha / \beta$ and $a$ is the constant of Theorem 2.9.

Proof. Let $\omega^{\prime}$ be the $F$-harmonic measure of $\partial B^{n}$ with respect to $B^{n} \backslash C$. Then [GLM 3, Lemma 3.18] yields for each $x \in B^{n} \backslash C$

$$
\omega^{\prime}(x) \leqq 4 \exp \left(-c(\alpha / \beta)^{1 / n} \int_{|x|}^{1} \frac{d t}{t}\right)=4|x|^{\varepsilon}
$$

where $\varepsilon=c(\alpha / \beta)^{1 / n} \leqq 1$ and $c>0$ depends only on $n$. This was proved in [GLM 3] under the additional assumption that $B^{n} \backslash C$ is a regular domain but since each component of $B^{n} \backslash C$ can be approximated from inside by regular domains and since the corresponding $F$-harmonic measures bound $\omega^{\prime}$ from above and satisfy (3.3), the inequality (3.3) for $\omega^{\prime}$ follows. Unfortunately, (3.3) does not immediately give (3.2) because the right side of (3.3) can be $\geqq 1$. To overcome this difficulty we first pick $r_{0} \in(0,1 / 2]$ such that $4 r_{0}^{\varepsilon}=1 / 2$, i.e.,

$$
r_{0}=(1 / 8)^{1 / \varepsilon} \text {. }
$$

Then $r_{0}$ depends only on $n$ and $\alpha / \beta$. Now (3.3) yields

$$
\omega^{\prime}(x) \leqq \frac{1}{2}
$$

for all $x \in B^{n}\left(r_{0}\right) \backslash C$. 
Next let $u$ belong to the upper class for $\omega$, see 2.1. Then $1-u$ is a sub-F-extremal in $B^{n} \backslash C$ and

$$
\varlimsup_{x \rightarrow y}(1-u(x)) \leqq \chi_{\partial B^{n}}(y)
$$

for all $y \in \partial\left(B^{n} \backslash C\right)$. The $F$-comparison principle, cf. [GLM 3, Lemma 2.3], yields

$$
1-\omega^{\prime} \leqq \omega
$$

in $B^{n} \backslash C$ and hence, by (3.5), $\omega(x) \geqq 1 / 2$ for all $x \in B^{n}\left(r_{0}\right) \backslash C$. To complete the proof let $\omega^{*}$ be the $F$-harmonic measure of $\partial B^{n}\left(r_{0}\right)$ with respect to the annulus $G=B^{n} \backslash \bar{B}^{n}\left(r_{0}\right)$. By Lemma 2.13

$$
\omega^{*}(x) \geqq b\left(r_{0}(1-|x|)\right)^{a}
$$

where $a$ and $b$ are the constants of Theorem 2.9. Finally, since $2 \omega \geqq \omega^{*}$ in $B^{n} \backslash\left(\bar{B}^{n}\left(r_{0}\right) \cup C\right)$, we obtain the desired inequality (3.2) from (3.6) with $c_{1}=b r_{0}^{a} / 2$ and the constant $a$ is the constant of Theorem 2.9. Note that in $B^{n}\left(r_{0}\right) \backslash C$ this inequality is trivial since $\omega \geqq 1 / 2$ and

there.

$$
b\left(r_{0}(1-|x|)\right)^{a} \leqq b r_{0}^{a} \leqq b / 2 \leqq 1 / 2
$$

3.7. Remarks. (a) It follows from Beurling's projection theorem, cf. the proof for Theorem 2.24, that for the ordinary plane harmonic measure $\omega$ in the situation of Theorem 3.1

$$
\omega(x) \geqq 1-\frac{4}{\pi} \arctan \sqrt{|x|} \geqq \frac{1}{\pi}(1-|x|)
$$

where the last inequality follows by elementary calculus. Simple examples based on non-lipschitzian quasiconformal mappings and the invariance property [GLM 2, Theorem 5.4] of $F$-harmonic measures show that in the general situation of Theorem 3.1 it is not possible to choose $a=1$ in (3.2), however, see Theorem 3.13 below.

(b) T. Carleman [C] proved the estimate (3.2) for the ordinary harmonic measure $\omega$ under the assumption that $C$ does not have compact components. He made use of the fact that in the plane the open set $B^{2} \backslash C$ then consists of simply connected domains. This allows the use of special conformal techniques, see [A, p. 42]. The corresponding result can be derived directly from Theorem 2.9 for general $F$-harmonic measures: If $C$ is a closed subset of $B^{n}$ without compact components and if $0 \in C$, then in $B^{n} \backslash C$

$$
\omega(x) \geqq b(1-|x|)^{a}
$$

where $\omega$ is the F-harmonic measure of $C$ with respect to $B^{n} \backslash C$ and $a$ and $b$ are the constants of Theorem 2.9 .

To prove (3.9) fix $x \in B^{n} \backslash C$. We may assume $x=t e_{n}, 0<t<1$. First note that

$$
k_{B^{n}}(x, C) \leqq-\log (1-|x|) .
$$


For this observe that since $0 \in C$,

$$
k_{B^{n}}(x, C) \leqq k_{B^{n}}(x, 0)
$$

and if $\gamma$ is the line segment $[0, x]$, then

$$
k_{B^{n}}(x, 0) \leqq \int_{\gamma} \operatorname{dist}\left(z, \partial B^{n}\right)^{-1} d s=-\log (1-|x|) .
$$

Next let $\gamma:[0,1] \rightarrow B^{n}$ be any rectifiable curve (parametrized by arc length) joining $x$ to 0 and let $\theta$ be the projection of $R^{n}$ onto $x_{n}$-axis. Then $\left|\theta^{\prime}\right| \leqq 1$ and $\gamma_{1}=\theta \circ \gamma$ is rectifiable and joins $x$ to 0 in $B^{n}$. Moreover, for $0 \leqq s \leqq 1$

and hence

$$
\operatorname{dist}\left(\gamma(s), \partial B^{n}\right) \leqq \operatorname{dist}\left(\gamma_{1}(s), \partial B^{n}\right)
$$

$$
\begin{aligned}
& \int_{\gamma} \operatorname{dist}\left(z, \partial B^{n}\right)^{-1} d s=\int_{0}^{1}\left|\gamma^{\prime}(s)\right| \operatorname{dist}\left(\gamma(s), \partial B^{n}\right)^{-1} d s \\
& \geqq \int_{0}^{1} \frac{\left|\theta^{\prime}(\gamma(s))\right|\left|\gamma^{\prime}(s)\right|}{\operatorname{dist}\left(\gamma_{1}(s), \partial B^{n}\right)} d s \geqq \int_{\gamma_{1}} \operatorname{dist}\left(z, \partial B^{n}\right)^{-1} d s \\
& \geqq-\log (1-|x|) .
\end{aligned}
$$

Thus $k_{B^{n}}(x, 0) \geqq-\log (1-|x|)$ and, by (3.12), $k_{B^{n}}(0, x)=-\log (1-|x|)$ and the inequality (3.10) follows from (3.11).

The inequality (3.9) now follows from (2.10) and (3.10).

If $F$ is the $n$-Dirichlet kernel, i.e., $F(x, h)=|h|^{n}$, then it is possible to derive a lower bound for $\omega$ as good as given by Beurling's projection theorem, see (3.8).

3.13. Theorem. Let $C$ be as in Theorem 3.1 and $F(x, h)=|h|^{n}$. If $\omega$ is the $F$-harmonic measure of $C$ with respect to $B^{n} \backslash C$, then for all $x \in B^{n} \backslash C$

$$
\omega(x) \geqq c_{2}(1-|x|)
$$

where $c_{2}>0$ depends only on $n$.

Proof. As in the proof for Theorem 3.1 we first show that

$$
\omega(x) \geqq \frac{1}{2}
$$

for $x \in B^{n}\left(r_{0}\right) \backslash C$ where $r_{0} \in(0,1 / 2]$ depends only on $n$ because $\alpha / \beta=1$ for this special $F$. Next let $A=B^{n} \backslash\left(\bar{B}^{n}\left(r_{0}\right) \cup C\right)$ and for $x \in A$ define

$$
v(x)=\log |x| /\left(2 \log r_{0}\right) \text {. }
$$

Then $v$ is an $F$-extremal in $A$ and at each point $y \in \partial A$

$$
\lim _{x \rightarrow y} v(x) \leqq \varliminf_{x \rightarrow y} u(x)
$$

where $u$ is any function in the upper class for $\omega$. Thus $\omega \geqq v$ in $A$ and by elementary calculus

$$
v(x) \geqq(|x|-1) /\left(2 \log r_{0}\right)
$$


in $A$ and the inequality (3.14) follows in $A$ with $c_{2}=-\left(2 \log r_{0}\right)^{-1}>0$. If $x \in \bar{B}^{n}\left(r_{0}\right) \backslash C$, then (3.14) follows from (3.15) because $r_{0} \leqq 1 / 2$ and hence

$$
c_{2}(1-|x|) \leqq(2 \log 2)^{-1}(1-|x|) \leqq(4 \log 2)^{-1}<\frac{1}{2} .
$$

The proof is complete.

3.16. Remark. The author conjectures that if $F(x, h)=|h|^{n}$ and if $C \subset \bar{B}^{n}$ is such that $C \cap B^{n}$ is closed in $B^{n}$, then

$$
\omega(x) \geqq \omega^{\prime}\left(|x| e_{1}\right)
$$

where $\omega$ is the $F$-harmonic measure of $C$ in $B^{n} \backslash C$ and $\omega^{\prime}$ is the $F$-harmonic measure of

$$
C^{\prime}=\left\{-t e_{1}: \partial B^{n}(t) \cap C \neq \emptyset, 0 \leqq t \leqq 1\right\}
$$

in $B^{n} \backslash C^{\prime}$, i.e. Beurling's projection theorem is true for this special $F$-harmonic measure. Note that Beurling's projection theorem is not needed for the estimate (3.14).

\section{Milloux's problem for quasiregular mappings}

Suppose that $f: G \rightarrow R^{n}$ is a $K$-quasiregular mapping, see [MRV], and that $|f| \leqq 1$. Let $C$ be a relatively closed subset of $G$. Then there is a kernel $F$ depending on $f$ such that $-\log |f(x)|$ is a super-F-extremal and $\beta / \alpha \leqq K^{2}$. For these results see [GLM 1-2]. Let $\omega$ be the $F$-harmonic measure of $C$ with respect to $G \backslash C$; set $\omega(x)=1$ for $x \in C$.

4.1. Lemma. If $|f(x)| \leqq m$ in $C$, then

$$
|f(x)| \leqq m^{\omega(x)}
$$

for all $x \in G$.

Proof. Cf. [GLM 2, Theorem 5.8]. Since $v(x)=-\log |f(x)|$ is a super-Fextremal in $G$ and since for all $y \in \partial(G \backslash C)$

$$
\varliminf_{x \rightarrow y} v(x) \geqq-(\log m) \chi_{c}(y),
$$

$v \geqq-(\log m) \omega$ in $G \backslash C$. Hence (4.2) holds in $G \backslash C$ and trivially in $C$.

4.3. Theorem. Suppose that $f$ is a $K$-quasiregular mapping of a proper subdomain $G$ of $R^{n}$ into $B^{n}$ and that $|f| \leqq m<1$ in the relatively closed subset $C \neq \emptyset$ of $G$ without compact components. Then

$$
|f(x)| \leqq m^{g(x)}
$$

where $g: G \rightarrow(0,1]$ is the function

$$
g(x)=\max \left(1-c k_{G}(x, C)^{\delta}, b \exp \left(-a k_{G}(x, C)\right)\right)
$$

and the positive constants $a, b, c$ and $\delta$ depend only on $n$ and $K$. 
Proof. The proof follows from Corollary 2.23, Lemma 4.1 and from the fact that $\beta / \alpha \leqq K^{2}$.

As above Theorem 3.1 and Lemma 4.1 yield

4.4. Theorem. Let $f$ be a $K$-quasiregular mapping of $B^{n}$ into $B^{n}$ such that $|f| \leqq m<1$ in the relatively closed subset $C$ of $B^{n}$ meeting each sphere $\partial B^{n}(t), 0 \leqq t<1$. Then for all $x \in B^{n}$

$$
|f(x)| \leqq m^{c(1-|x|)^{a}}
$$

where the positive constants $a$ and $c$ depend only on $n$ and $K$.

\section{References}

[A] Ahlfors, L. V.: Conformal invariants. Topics in geometric function theory. - McGrawHill Book Company, New York, 1973.

[B] BeuRling, A.: Études sur un problème de majoration. - Thèse, Uppsala, 1933.

[C] Carleman, T.: Sur les fonctions inverses des fonctions entières d'ordre fini. - Ark. Mat. Astr. Fys. 15: 10, 1921.

[GP] Gehring, F. W., and B. PALKa: Quasiconformally homogeneous domains. - J. Analyse Math. 30, 1976, 172-199.

[GLM 1] Granlund, S., P. Lindevist, and O. Martio: Conformally invariant variational integrals. - Trans. Amer. Math. Soc. 277, 1983, 43-73.

[GLM 2] Granlund, S., P. Lindevist, and O. Martio: F-harmonic measure in space. - Ann. Acad. Sci. Fenn. A I Math. 7, 1982, 233-247.

[GLM 3] Granlund, S., P. LindQvist, and O. Martio: Phragmén-Lindelöf's and Lindelöf's theorems. - Ark. Mat. 23, 1985, 103-128.

[HM] HeINonen, J., and O. MARTio: Estimates for $F$-harmonic measures and Øksendal's theorem for quasiconformal mappings. - To appear.

[LM] LINDQVIST, P., and O. MARTIO: Regularity and polar sets for supersolutions of certain degenerate elliptic equations. - To appear.

[MRV] MARTIO, O., S. RICKMAN, and J. VÄISÄLÄ: Definitions for quasiregular mappings. - Ann. Acad. Sci. Fenn. A I Math. 448, 1969, 1-40.

[M] MAZ'JA, V. G.: The continuity at a boundary point of the solutions of quasilinear elliptic equations. - Vestnik Leningrad. Univ. 25: 13, 1970, 42-55 (Russian).

[R] RICKMAN, S.: On the number of omitted values of entire quasiregular mappings. - J. Analyse Math. 37, 1980, 100-117.

[V 1] VuORINEN, M.: Capacity densities and angular limits of quasiregular mappings. - Trans. Amer. Math. Soc. 263, 1981, 343-354.

[V 2] Vuorinen, M.: On the Harnack constant and the boundary behavior of Harnack functions. - Ann. Acad. Sci. Fenn. A I Math. 7, 1982, 259-277.

University of Jyväskylä

Department of Mathematics

SF-40100 Jyväskylä

Finland

Received 28 May 1986 